\title{
Noise induced re-entrant transitions in exciton bistable system
}

\author{
Yu. Gudyma*, B. Ivans'kii, O. Semenko \\ Yu. Fed'kovich Chernivtsi National University \\ 2, Kotsyubinsky str., 58012 Chernivtsi, Ukraine \\ *E-mail:yurig@ukr.net
}

\begin{abstract}
We study a re-entrant transition in an exciton bistable system under the influence of multiplicative noise. The re-entrant behavior is predictable for specific values of the system control parameter when increasing the multiplicative noise intensity. The system of Wannier-Mott excitons exhibits bistable $\leftrightarrow$ monostable transitions in a window of intermediate amplitudes of the multiplicative noise intensity demonstrating its destructive and constructive role.
\end{abstract}

Keywords: exciton, resonance absorption, multiplicative noise, transition.

Manuscript received 10.11.05; accepted for publication 15.12.05.

\section{Introduction}

Systems with a definite number of competing states of local stability play a leading role in the contemporary analysis of various physical phenomena and their possible applications. In a growing number of cases such situations occur in open systems, i.e., systems that require continual fluxes of the energy or matter. Our focus will be on the dynamics of solid-state WannierMott excitons with bistable behavior. Due to an excitonic resonance, an intrinsic absorption optical bistability may occur in a narrow spectral range beyond the critical light intensity. An external feedback in the form of optical resonator is not necessary for such bistability.

It has been found experimentally that resonatorless optical bistability near the exciton resonance takes place at rather small exciton concentrations $\left(n_{\mathrm{ex}} \sim 10^{15} \mathrm{~cm}^{-3}\right)$ and the intensity of incident radiation $1 \mathrm{~kW} / \mathrm{cm}^{2}$ at room temperatures $[1,2]$. It is important that optical nonlinear effects occur during resonance formation of excitons at a significantly lower incident-light intensity as compared with excitation of isolated atoms. The exciton nonlinearities leading to this bistability has been investigated theoretically in early papers [3, 4]. It has been found that the concentration of excitons induced by resonance light of a sufficiently large intensity can be an ambiguous function of that intensity, if the width and position of the absorption line depends on the concentration. In fact, the increase of external radiation leads to growth of the density of exciton states and raising exciton-exciton collision role. In this case, the response of the system depends on the number of excitons induced by external radiation. Thus, an increase of the light intensity induces the change of optical characteristics in semiconductors [5]. The optical device based on such semiconductors has not been already designed, but the latter phenomenon has a fundamental meaning in the study of non-equilibrium systems.

Hence, for the correct description of the position and linewidth of the exciton, it is impossible to neglect many-body effects [6]. The exciton-density dependence of the exciton resonance position has been intensely investigated by spectrally resolved four-wave mixing [7, 8]. Furthermore, a sophisticated case of the energy renormalization of the biexciton resonance in $\mathrm{ZnSe}$ quantum wells takes place [9]. These examples indicate the importance of taking into consideration the dependence of the resonance frequency on the concentration of excitations in various systems.

The processes of creation and transformation of an ordered structure far from equilibrium are similar to phase transitions in an equilibrium system. Noise induced phenomena associated with kinetic transitions have special place in non-equilibrium processes [10] and have been actively discussed [11-13]. It has become apparent that even small noise can induce qualitative changes in the system far from thermal equilibrium [14].

In this work, the optical bistable system near the excitonic resonance is studied in the presence of the multiplicative noise. We predict the appearance of noise induced re-entrant transitions in the narrow region of light intensities. The bifurcation diagrams showing that region were obtained by numerically calculating the extremum of the probability for the stationary density of excitons in bistable semiconductor.

The paper is organized as follows. In the next Section, we briefly discuss the basic equations for the 
exciton bistable system under some multiplicative noise. The analysis of its stochastic behavior and re-entrant transitions are presented in the third Section. Finally, several conclusions have been given in Sec.IV.

\section{Basic equations}

Transport equations for the laser radiation with the intensity $I(z)$ and quasi-particle concentration $n(z, t)$ can be written in the form

$\frac{d I}{d z}=-\alpha(\omega, n) I(z)$

$\frac{\partial n}{\partial t}=D \frac{\partial^{2} n}{\partial z^{2}}+\alpha(\omega, n) I(z)-\frac{n}{\tau}$,

where $\alpha(\omega, n)$ and $D$ are the light absorption and quasi-particle diffusion coefficients, respectively; $\tau$ is the quasi-particle lifetime. The surface $z=0$ of a semiconductor plate is illuminated with a light beam of the intensity $I_{0}$. In equation (2), we will confine ourselves to a linear function for the recombination concentration of excitons.

The frequency dependence of the light-absorption coefficient $\alpha(\omega)$ for the resonance light absorption is given by

$\alpha(\omega, n)=\alpha_{0} \quad\left\{1+\left(\frac{\omega_{r}^{\prime}-\omega}{\Delta \omega}\right)^{2}\right\}^{-1}$

Collective interactions (interactions of excitons with lattice or with each other, exchange interactions, etc.) in the exciton system at high excitation levels cause the resonance frequency $\omega_{r}^{\prime}$ to become a function of the excitation concentration that decreases with $n$ : $\omega_{r}^{\prime}(n)=\omega_{r}-a n$. This behavior is due to effective attraction of excitons. The magnitude of the lightabsorption coefficient (3) at fixed frequencies depends essentially on the resonance frequency. In the case of excitons, $\Delta \omega$ and $a n$ are the width and shift (in the linear approximation of the quasi-particle concentration) of the exciton level $\omega_{r}$. The concentration-induced blue shift and the broadening of the exciton absorption line increase absorption in the relation (3).

Assuming that the diffusion length exceeds the plate thickness and introducing the averaged light intensity, we obtain a nonlinear concentration balance equation for a system of Wannier-Mott excitons

$\frac{d n}{d t}=I_{0} l^{-1}\left\{1-\exp [-\alpha(\omega, n) l\}-n \tau^{-1}\right.$.

The macroscopic equation (4) in the frequency region $\omega \leq \omega_{r}$ has from one to three solutions depending on the value of the control parameter. In the last case, this leads to a hysteresis in the quasi-particle distribution that is determined by the laser-radiation intensity. This results from a bell shape (pseudoLorentzian form) of the absorption factor as a function of the exciton concentration in a certain frequency region. For this reason, the phenomena of resonatorless optical bistability takes place in the exciton frequency region. An internal feedback in the system provides a balance condition between the processes of exciton generation and recombination. Toyozawa [3] examined similar nonlinear situation for the system with threshold behavior of absorption.

Having a fixed incident light frequency, we can change over to dimensionless variables in Eq. (4):

$\frac{d \eta}{d \theta}=\beta\left\{1-\exp \left[-\lambda /\left(1+\Omega(1-\eta)^{2}\right)\right]\right\}-\eta \equiv f(\eta)$

with $\quad \theta=t / \tau, \quad \lambda=\alpha_{0} l, \quad \Omega=\left(\left(\omega_{\mathrm{r}}-\omega\right) / \Delta \omega\right)^{2}$, $\eta=a n /\left(\omega_{r}-\omega\right), \quad \beta=a \tau I_{0} /\left(\omega_{\mathrm{r}}-\omega\right) l$. By mechanical analogy Eq. (5) describes an over-damped oscillatory system, while the magnitude $f(\eta)$ corresponds to external forces.

\section{Re-entrant transitions}

Let us examine the changes in the states of the system induced by fluctuations of the light intensity. For the system of excitons, the light intensity is an external parameter that appears in Eq. (5) as a multiplicative factor. We shall describe the random breakdown of light coherence by the following stochastic process $\beta(t)=\beta+\sigma \xi(t)$, where the external noise $\xi(t)$ is characterized by very rapid fluctuations (Gaussian white noise) to compare with the characteristic evolution time of system. This means that the one-dimensional variable $\eta$ undergoes the influence of a random external perturbation. The intrinsic fluctuations of the system are not considered, since they do not induce transitions [10]. These fluctuations (both additive and multiplicative) only shift the region of multiple quasi-particle distribution towards a major value of the control parameter.

In terms of generalized functions, the Gaussian white noise is a derivative of the Wiener process. Therefore, Eq. (5) is a stochastic differential equation in the sense of Stratonovich associated with the Fokker-Plank equation that defines the evolution of the transition probability $p\left(\eta, \theta / \eta^{\prime}, \theta^{\prime}\right)[10]$

$$
\begin{aligned}
& \frac{\partial p\left(\eta, \theta \mid \eta^{\prime}\right)}{\partial \theta}=-\frac{\partial}{\partial \eta}\left[A(\eta, \theta) p\left(\eta, \theta \mid \eta^{\prime}\right)\right]+ \\
& +\frac{1}{2} \frac{\partial^{2}}{\partial \eta^{2}}\left[B(\eta, \theta) p\left(\eta, \theta \mid \eta^{\prime}\right)\right]
\end{aligned}
$$

where 


$$
\begin{aligned}
& A(\eta, \theta)=\beta\left\{1-\exp \left[-\lambda /\left(1+\Omega(1-\eta)^{2}\right)\right]\right\}-\eta+ \\
& +\frac{\sigma^{2}}{2} \Omega \lambda \frac{1-\eta}{1+\Omega(1-\eta)^{2}} \exp \left[-\lambda /\left(1+\Omega(1-\eta)^{2}\right)\right] \times \\
& \times\left\{1-\exp \left[-\lambda /\left(1+\Omega(1-\eta)^{2}\right)\right]\right\}, \\
& B(\eta, \theta)=\sigma^{2}\left\{1-\exp \left[-\lambda /\left(1+\Omega(1-\eta)^{2}\right)\right]\right\}^{2} .
\end{aligned}
$$

The states of system can be adequately described by the stationary probability density $p(\eta)$, if not to consider the microscopic transients of a nonlinear system with respect to an external noise. The stationary solution of the homogeneous Fokker-Plank equation (6)

$$
\begin{aligned}
& p_{s}(\eta)=N\left\{1-\exp \left[-\lambda /\left(1+\Omega(1-\eta)^{2}\right)\right]\right\}^{-1} \times \\
& \times \exp \left(-\frac{2}{\sigma^{2}} \int_{0}^{\eta}\left[\beta\left\{1-\exp \left[-\lambda /\left(1+\Omega(1-U)^{2}\right)\right]\right\}-U\right] /\right. \\
& \left./\left[1-\exp \left[-\lambda /\left(1+\Omega(1-U)^{2}\right)\right]\right]^{2} d U\right)
\end{aligned}
$$

corresponds to the diffusive stochastic process. Here, $N$ is a constant to be determined from the the normalization condition

$$
\int_{0}^{b} p_{s}(\eta) d \eta=1
$$

The limits of integration are given by physical restrictions of the problem, i.e., by variation of the exciton concentration from zero to that at which the Bose condensation takes place.

The solution (7) can be written by analogy with the well-known canonical distribution of equilibrium thermodynamics

$$
p_{s}(\eta)=N \exp \left(-2 V(\eta) / \sigma^{2}\right) \text {. }
$$

Here $V(\eta)$ is the effective potential. The random diffusion process $\eta(\theta)$ is ergodic, since the probability density (9) is normalized. It follows from the fact that the product $p_{s}(\eta) d \eta$ is the time over which the arbitrary trajectory of the diffusion process approaches the point $\eta$ at infinity. Thus, the maxima of the probability density correspond to stable stationary states in which the system dwells relatively long, while the minima correspond to unstable stationary states that are passed very quickly. The macroscopic stationary states of the system can be found from extremum conditions of the stationary probability density [10]. Noise induced transitions appear when the density function changes from unimodal into bimodal distribution. Thus, for examination of long-time system behavior, it is sufficient to study the extremes of the effective potential $V(\eta)$, since the neighborhood of potential minima of the $\sigma^{2}$ order gives the basic contribution in the stationary probability density. A numerical analysis is carried out for the model of illuminated CdS-like semiconductor with the following parameters: $\tau=10^{-3} \mathrm{~s}$, $a=2.5 \cdot 10^{-7} \mathrm{~cm}^{3} / \mathrm{s}, \Omega=100, \lambda=0.055$. The level of the light flux intensity is $10^{20}$ photon $/ \mathrm{cm}^{2} \cdot \mathrm{s}$.

Let us consider the behavior of the system for supercritical values of the control parameter $\beta_{c}=18.64$.

In the absence of external multiplicative noise, the system is characterized by one stationary state near the zero value of $\eta$ (Fig. 1, curve marked as circles), which corresponds to the shifted resonance frequency. The inset shows the function near the zero line. But the growth of fluctuations of the incident light, which are characterized by the noise amplitude $\sigma$ or dispersion $\sigma^{2}=0.09$, leads to the appearance of bistability in the concentration dependence (Fig. 1, curve marked as squares). Hence, the given qualitative reconfiguration of the dynamical system regime with resonance absorption of light should be treated as an external noise induced bistability of exciton states below the critical intensity of the incident radiation. By the analogy with the motion of particle in the classic potential $V(\eta)$, this mean that small external noises transform an infinite motion of particle into finite motion in a certain interval. On the other hand, for supercritical values of the controlling parameter $\beta$, the external noise shifts the region of bistability towards the higher values of the incident light intensity (suppression of bistability by noise). As an example, we illustrate this behavior in Fig. 2.

Hence, external white multiplicative noise has a contradictory role in the open nonlinear system. It leads to enlargement of the system bistability region and its shift. Noise may lead to a bistable behavior in systems where this phenomenon cannot take place without noise. On the other hand, it can suppress the states that are already present in the deterministic system. Such

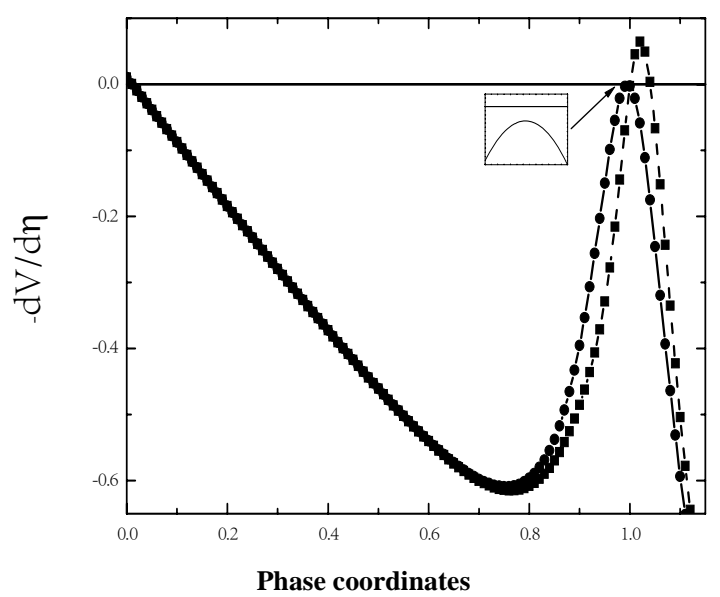

Fig. 1. "Stochastic force" plotted versus phase coordinates for different values of noise amplitude $\sigma$. The line with circles corresponds to $\sigma^{2}=0$, and another one (with squares) to $\sigma^{2}=$ $=0.09$. 


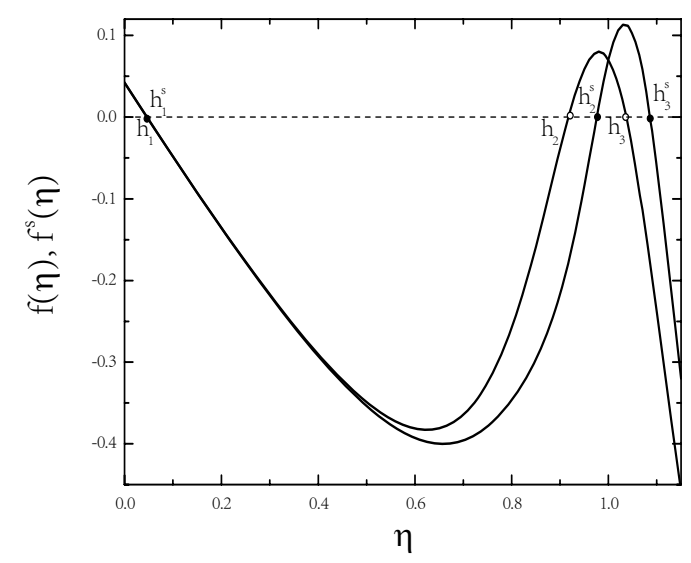

Fig. 2. Comparison of deterministic $f(\eta)$ and stochastic $f^{s}(\eta)$ "forces" for $\beta=20, \Omega=25$. The amplitude of the multiplicative noise intensity is $\sigma=7$.

combination of destructive and constructive nature of external multiplicative noise may lead to unusual behavior of the system at the re-entrant transition. In this case, the noise-induced transition brings the system to bimodality; while the re-entrant transition takes places at a higher value of the multiplicative noise intensity, making the system to be unimodal again. The system exhibits a suppression bistability, followed by the reentrant transition back to bistability, both induced by multiplicative noise. In other words, the system undergoes a suppression of bistability in a narrow window of intermediate intensities. It will be shown that our prediction takes place. For this purpose, we will estimate the critical value of the light intensity by changing the amplitude of the multiplicative noise

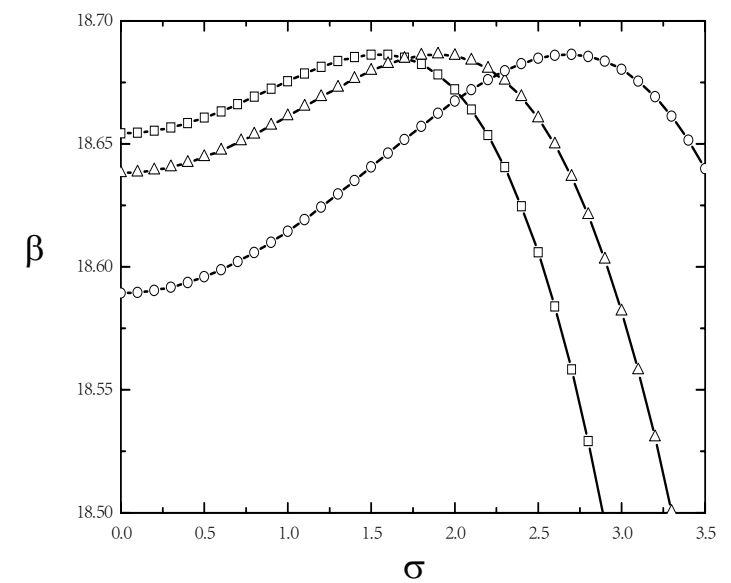

Fig. 3. Phase diagram of the system (dependence of the critical value of the nondimensional laser radiation intensity $\beta$ versus the amplitude of the multiplicative noise intensity $\sigma$ for various values of $\Omega$. The value $\Omega=50$ corresponds to the lines with circles, $\Omega=100$ to the lines with triangles and $\Omega=150$ to the lines with squares. intensity. In order to illustrate the behavior of an optical bistable system being under excitonic resonance, we have numerically examined the extremum of the effective potential (9).

As one can see from Fig. 3, the $\beta_{c}$ (critical value of incident radiation intensity) depends on the parameter $\Omega$ which is the ratio between the value of detuning at the excitonic resonance and excitonic line width. In the deterministic case $(\sigma=0)$, an increase of the parameter $\Omega$ requires higher intensities of light $\beta$ for bistability to take place in the exciton system. Probability distributions are bimodal for points above the curves and unimodal below. The growing multiplicative noise increases the critical value of the laser radiation intensity at first, but than decreases it abruptly at larger values of $\sigma$. At the higher then the critical value of the light intensity, a crossover takes place from the bistable to monostable region and back to bistable while increasing the noise amplitude. The latter re-entrant phenomenon is accompanied with the shift of the transition line.

Note that the described phenomena resemble the appearance of stochastic resonance since constructive manifestation of noise is optimal for the intermediate value of the multiplicative noise amplitude. Previous order resumes for a larger value of the noise intensity amplitude showing its destructive role. It can be easily seen from Fig. 4 that the latter behavior takes place for a narrow window of values of the control parameter $\beta$.

The stochastic system exhibits re-entrant behavior as a function of multiplicative Gaussian white noise. Besides, an increase of noise induces bistability for smaller values of laser irradiation than in the deterministic case. As discussed in paper [15], nonlinear systems can undergo noise induced re-entrant transitions also under the influence of colored noises.

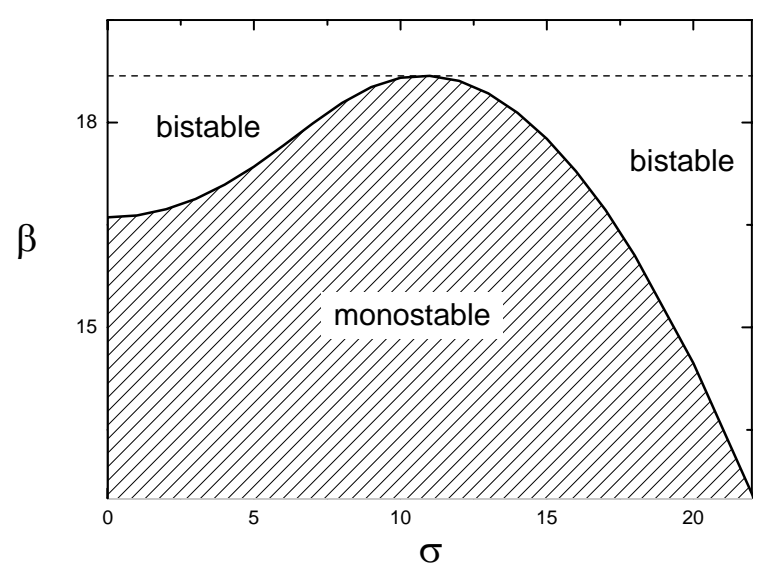

Fig. 4. Phase diagram of the system here is the dependence of the critical value for the nondimensional laser radiation intensity $\beta$ versus the amplitude of the multiplicative noise intensity $\sigma$. The dotted lines marks the region of the re-entrant transition. 
Quasi-white noise approach uses assumption that the width of the noise spectrum $(\sim \sigma)$ is by far larger than the inverse time of passing to a steady regime. Another simplification used in this approach is neglecting the internal structure of a laser beam. Qualitative results of the research doesn't change, if take into account the described structure.

\section{Conclusions}

In this paper, the influence of multiplicative noise on a system of Wannier-Mott excitons have been examined. We have presented the evidence of the re-entrant transition in the bistable model on excitonic resonance. The re-entrant transition is induced by parametric noise of the laser radiation intensity, which demonstrates the dual role of noise amplitude as a control parameter. It has been found that even a sufficiently small external multiplicative noise shifts the region of bistability towards the higher values of the incident light intensity, in other words, noise suppresses the bistability. But the further increase of noise induces the bistability of the stochastic system for smaller values of laser irradiation than in the deterministic case.

We have numerically constructed the transition line diagrams, i.e., the boundaries of the bimodal-unimodal transitions. The form and the position of the lines on the plane $\{\beta, \sigma\}$ depends on the ratio between the value of detuning at the excitonic resonance and excitonic line width. The state diagrams show that the system passes the monostable region in a window of intermediate amplitudes of the multiplicative noise intensity for the specific value of $\beta$. The transition is re-entrant, i.e., bistability is resumed at a higher noise intensity. An immediate cause of re-entrant behavior is the resonance origin of the excitonic absorption mechanism in semiconductors. Thus, one can expect the appearance of the latter phenomenon in other similar systems with the resonance absorption.

\section{References}

1. H. Rossmann, F. Henneberger, and J. Voigt, Memory effect in the excitonic transmission of CdS // Phys. status solidi (b) 115(1), p. K63-K67 (1983).
2. F. Henneberger, Optical bistability at the absorption edge of semiconductors // Ibid. 137(2), p. 371-432 (1986).

3. Y. Toyozawa, Population instability and optical anomalies in high density excited system // Solid State Communs 28(7), p. 533-536 (1978).

4. Y. Toyozawa, Bistability and anomalies in absorbtion and resonance scattering of intense light // Ibid. 32(1), p. 13-18 (1979).

5. H.M. Gibbs, Optical bistability: Controlling light with light. Academic Press, Orlando (1985).

6. G. Manzke, Q.Y. Peng, K. Henneberger, U. Neukirch, K. Hauke, K. Wundke, J. Gutowski, and D. Hommel, Density dependence of the exciton energy in semiconductors // Phys. Rev. Lett. 80(22), p. 4943-4946 (1998).

7. S. Hataoka, A. Itoh, I. Tanahashi, and K. Tanaka, High-density excitation effects of excitons in $\mathrm{ZnSe}$ quantum wells by spectrally resolved four-wave mixing // Journal of Luminescence 87-89, p. 853-855 (2000).

8. S. Wachter, M. Maute, H. Kalt, I. Galbraith, C. Sieh, T. Meier, and S. W. Koch, Excitation induced shift and broadening of the exciton resonance // Physica B 314(1-4), p. 309-313 (2002).

9. M. Maute, S. Wachter, H. Kalt, K.Ohkawa, and D. Hommel, Energy renormalization and binding energy of the biexciton // Phys.Rev.B 67, 165323 (2003).

10. W. Horsthemke, and R. Lefever, Noise-induced transitions. Springer, Berlin (1984).

11. M.S. Miguel, and R. Toral, Stochastic effects in physical systems / In: Instabilities and nonequilibrium structures VI. Kluwer Academic Publishers, Dordrecht (2000), p. 35-130.

12. J.M. Sancho, and J. Garcia-Ojalvo, Noise-induced order in extended systems: a tutorial / In: Stochastic processes in physics, chemistry and biology. Springer, Berlin (2000), p. 235-246.

13. Yu.V. Gudyma, Nonequilibrium first-order phase transition in semiconductor system driven by colored noise // Physica A 331(1-2), p. 61-68 (2004).

14. P.S. Landa, and P.V.E. McClintock, Changes in the dynamical behavior of nonlinear systems induced by noise // Phys. Repts 323, p. 1-80 (2000).

15. H.S. Wio, and R. Toral, Effect of non-Gaussian noise sources in a noise induced transition // Physica D 193(1-4), p. 161-168 (2004). 